\title{
Low prevalence of neuropathic-like pain symptoms in long-term controlled acromegaly
}

\author{
Victoria R. van Trigt ${ }^{1} \cdot$ Iris C. M. Pelsma ${ }^{1}$ (D) Herman M. Kroon ${ }^{2} \cdot$ Alberto M. Pereira $^{1}$. Coen van der Meulen ${ }^{3}$. \\ Margreet Kloppenburg ${ }^{3,4} \cdot$ Nienke R. Biermasz $^{1} \cdot$ Kim M. J. A. Claessen $^{1}$
}

Accepted: 4 October 2021 / Published online: 23 October 2021

(c) The Author(s) 2021

\begin{abstract}
Purpose Pain is a common symptom of acromegaly, impairing health-related quality of life (HR-QoL) significantly despite long-term disease remission. Neuropathic-like pain (NP-like) symptoms are invalidating, with great impact on HR-QoL. Studies characterizing or investigating the etiology of pain in acromegaly are scarce. Therefore, we aimed to assess NP-like symptoms in a cohort of controlled acromegaly patients.

Methods Forty-four long-term controlled acromegaly patients (aged 62.6 \pm 12.6 years; $56.8 \%$ female) were included in this cross-sectional study. NP-like symptoms were assessed using the validated painDETECT questionnaire. Patients were divided in three probability-based NP-like symptoms categories based on the total score (range $0-35)$ : unlikely $(\leq 12)$, indeterminate (13-18) and likely ( $\geq 19)$. HR-QoL (physical component score (PCS), and mental component score (MCS)), and self-reported pain were assessed using Short Form-36 (SF-36). Potential risk factors were determined using linear regression analyses. Results Self-reported pain was reported by 35 patients (79.5\%). Likely NP-like symptoms were present in 4/44 patients $(9.1 \%)$, and indeterminate NP-like symptoms in 6/44 patients (13.6\%). All patients with likely NP-like symptoms were female. Higher painDETECT scores were negatively associated with HR-QoL (PCS: $r=-0.46, P=0.003$; MCS: $r=-0.37$, $\mathrm{P}=0.018)$, and $\mathrm{SF}-36$ pain scores $(\mathrm{r}=-0.63, \mathrm{P}<0.0001)$. Female sex was a risk factor for NP-like symptoms.

Conclusions Pain was prevalent in controlled acromegaly patients, whereas NP-like symptoms were relatively infrequent, and only observed in females. NP-like symptoms were associated with lower HR-QoL in acromegaly. Since specific analgesic therapy is available, awareness for characterization, increased understanding, and clinical trials regarding neuropathic pain identification and treatment in acromegaly patients are warranted.
\end{abstract}

Keywords Acromegaly $\cdot$ GH/IGF-1 Pain · Neuropathic pain $\cdot$ HR-QoL

Victoria R. van Trigt and Iris C. M. Pelsma: Shared first authors

Iris C. M. Pelsma

I.C.M.Pelsma@lumc.nl

1 Department of Medicine, Division of Endocrinology, and Center for Endocrine Tumors Leiden, Leiden University Medical Center, Albinusdreef 2, 2333 ZA Leiden, The Netherlands

2 Department of Radiology, Leiden University Medical Center, Leiden, The Netherlands

3 Department of Rheumatology, Leiden University Medical Center, Leiden, The Netherlands

4 Department of Epidemiology, Leiden University Medical Center, Leiden, The Netherlands

\section{Introduction}

Acromegaly is associated with an abundance of partially irreversible symptoms and comorbidities, significantly contributing to decreased health-related quality of life (HR-QoL) [1], which improves after acromegaly treatment albeit without complete normalization [2]. Pain is a prominent and invalidating feature-self-reported by $73 \%$ of patients 10 years after initial therapy [3-5] —reported more frequently compared to age-, and gender-matched controls [6]. Several acromegaly-associated musculoskeletal comorbidities may cause pain, e.g. carpal tunnel syndrome (prevalence: $18-84 \%$ ) [7, 8], arthropathy characterized by cartilage hypertrophy and severe osteophytosis (prevalence: 72-94\%) [1, 9-11], and vertebral fractures (prevalence: 60\%) [12-14]. Additionally, headaches are frequently reported by patients 
due to either mechanical adenoma pressure, or growth hormone $(\mathrm{GH})$ excess (prevalence: $37-87 \%$ ) [8, 15].

Considering the diverse etiology of pain in acromegaly patients, the origin, and type of pain are difficult to determine. Generally, pain can be divided into two types: nociceptive pain caused by activation of nociceptors, and neuropathic pain resulting from lesions/diseases of the peripheral somatosensory nervous system [16]. Neuropathic pain is considered more invalidating than nociceptive pain [17, 18], being associated with depression and lower HR-QoL [18]. Moreover, several acromegaly-associated comorbidities might increase the risk for neuropathic pain, e.g. diabetic neuropathy, and mechanical complaints (incl. compression neuropathies) $[4,7,8,19-21]$. Since specific analgesic therapy, e.g. tricyclic antidepressants, is indicated for neuropathic pain, adequate neuropathic pain identification is warranted to optimally address pain in patients with acromegaly [22, 23].

To our knowledge, only one previous study investigated neuropathic pain in pituitary adenoma patients, including acromegaly, of whom $20 \%$ reported neuropathic-like pain symptoms [3]. In the present study, we investigated the prevalence of neuropathic-like pain symptoms, potential associated clinical determinants, and the relationship between neuropathic-like pain symptoms and HR-QoL in a cohort of controlled acromegaly patients.

\section{Materials and methods}

\section{Study design and patient selection}

\section{Study design}

All patients gave informed consent for this cross-sectional observational study, which was approved by the Medical Ethics Committee. A standardized questionnaire on medical history, and current health condition was completed, including the past and current use of medication. Serum samples were obtained to assess pituitary function. Validated questionnaires, including a specific neuropathic pain symptom questionnaire, were filled out (vide infra).

\section{Patients}

Forty-four patients with well-controlled acromegaly with complete neuropathic pain questionnaires were included in the present study. Twenty-five patients in remission that participated in our long-term follow-up study on musculoskeletal acromegalic complications (using data from the last study visit—details published prior [13, 24]) were combined with nineteen additional patients in remission that were consecutively included at the time of the last study visit from new referrals to our expertise center.

\section{Study parameters}

\section{Acromegaly disease parameters and pituitary function}

Clinical assessment of patients with acromegaly has been described previously [1,9-11, 25-31]. Briefly, disease remission was defined as normal glucose-suppressed serum GH and IGF-1 levels (corrected for age and sex) [1, 9-11, 16, 25-31]. Hypopituitarism was treated according to available guidelines [13, 24, 32].

\section{Assays}

Serum GH levels were measured using a nationally harmonized assay (IDS-iSYS immunoanalyzer; harmonization factor: 1.02) [33]. For IGF-1, serum levels (IDS-iSYS immunoanalyzer), and SDS scores were used (based on age- and sex-dependent $\lambda-\mu-\sigma$ smoothed reference curves) $[34,35]$.

\section{Questionnaires}

Patients filled out all questionnaires based on their health status at the moment of the study visit. Notably, because of the observational nature of the study, the current prescribed treatments, and medications were continued.

Neuropathic pain The 9-item painDETECT Questionnaire (painDETECT) was validated to identify neuropathic-like pain symptoms in various conditions including lower back pain, rheumatoid arthritis, and primary OA [36-41]. The painDETECT questionnaire (in English) is shown in Supplementary File 1. The painDETECT consists of seven questions on different aspects of neuropathic pain (7-item version), and two additional questions on pain localization and course over time (9-item version) (vide infra). Examples of questions are "Do you have a tingling or prickling sensation in the area of your pain (like crawling ants or electrical tingling)?", and "Does slight pressure in this area, e.g. with a finger, trigger pain?". Total painDETECT scores based on the 7-item version of the painDETECT ranged from 0 to 35 (7 questions using a 6-point Likert scale from 0 to 5) [42]. Based on the total painDETECT scores, patients were divided into three groups, reflecting the probability of neuropathic-like pain symptoms: unlikely-NP, total score $\leq 12$; indeterminate-NP, total score 13-18; likely-NP, total score $\geq 19$ [43, 44]. Outcomes of the remaining two questions of the 9-item painDETECT on course of pain over time, and pain radiation were available for a subset of 35 , and 37 patients, respectively. 
Additionally, information on pain localization was extracted from the drawings of individual patients on the human figure within the painDETECT questionnaire. Data were available for 31 patients. Pain localization was divided into 4 categories: (1) pain in one specific location, (2) pain in one limb, (3) pain in two separate limbs, and (4) pain diffusely spread throughout the body (Fig. 1).

Joint symptoms All patients completed the following questionnaires: Australian/Canadian Osteoarthritis Hand Index (AUSCAN) to measure hand disability [45]; Disabilities of the Arm, Shoulder and Hand (DASH) to assess disability of the upper limb [46-48]; Western Ontario and McMaster Universities Arthritis Index (WOMAC) to evaluate lower limb joint symptoms [45, 49]. In detail, the AUSCAN questionnaire [45] —used for evaluation of hand symptoms in the last $48 \mathrm{~h}$-rates all items on a 5-point Likert scale ranging from 0 (none) to 4 (extreme), and divided items in three categories: pain (scores ranging from 0 to 20), stiffness (range $0-4$ ), and joint function (range 0-36), with total AUSCAN scores ranging from 0 to 60 . The DASH questionnaire [46$48,50]$ — used to assess symptoms and function of the upper limbs in the last 7 days-rates 30 questions on a 5-point Likert scale. Following transformation, total scores ranged from 0 to 100. The WOMAC questionnaire [51]—used to assess disability of the lower limb-rates all items on a $100 \mathrm{~mm}$ visual analog scale (VAS), with 0 representing the absence of complaints, and 100 the worst score possible. Total WOMAC scores ranged from 0 to 300 , with subscores (pain, stiffness, and function) ranging from 0 to 100 . For all questionnaires, higher total scores indicated greater disability.
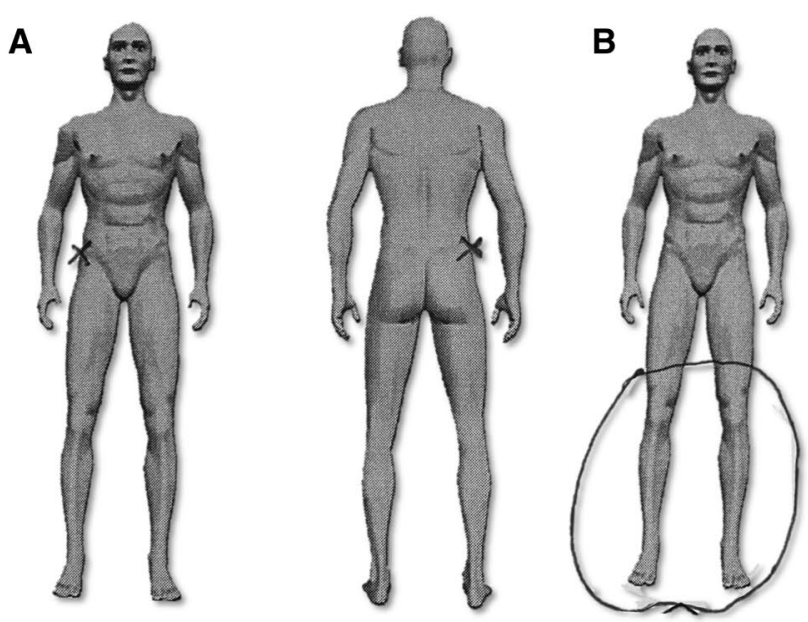

Fig. 1 Examples of different categories of pain localization based on drawings in controlled acromegaly patients. Examples of the pain localization as assessed by drawings of individual controlled acromegaly patients on the human figure on the painDETECT question-
Health-related QoL Patients completed the HR-QoL questionnaire Short Form-36 (SF-36) [52]. Physical health component score (PCS), mental health component scores (MCS), and pain component scores were calculated [53], with higher scores indicating better HR-QoL (total score 0-100) [54]. Based on the SF-36 question on presence of pain (ranging from absence to very severe), current presence of pain was defined as any answer but absence (dichotomous).

\section{Statistical analysis}

SPSS for Windows version 25.0 (SPSS Inc., Chicago, IL, USA) was used for data analysis. Data are presented as N (\%), mean $\pm \mathrm{SD}$, or median (IQR). Pearson's correlation analyses and linear regression analysis using numerical 7-item painDETECT total scores were performed. P-values $<0.05$ were considered significant.

\section{Results}

\section{Patient characteristics}

Forty-four patients with controlled acromegaly (mean age $62.6 \pm 12.6$ years, $56.8 \%$ female) were included, as summarized in Table 1. Patients were in remission for a median of 17.1 years (IQR 7.3-25.4), with 18 patients currently receiving pharmacological treatment. Ten patients received somatostatin (SMS) analogue monotherapy, three patients received Pegvisomant (PegV) monotherapy, three patients were treated with SMS analogue and PegV combination
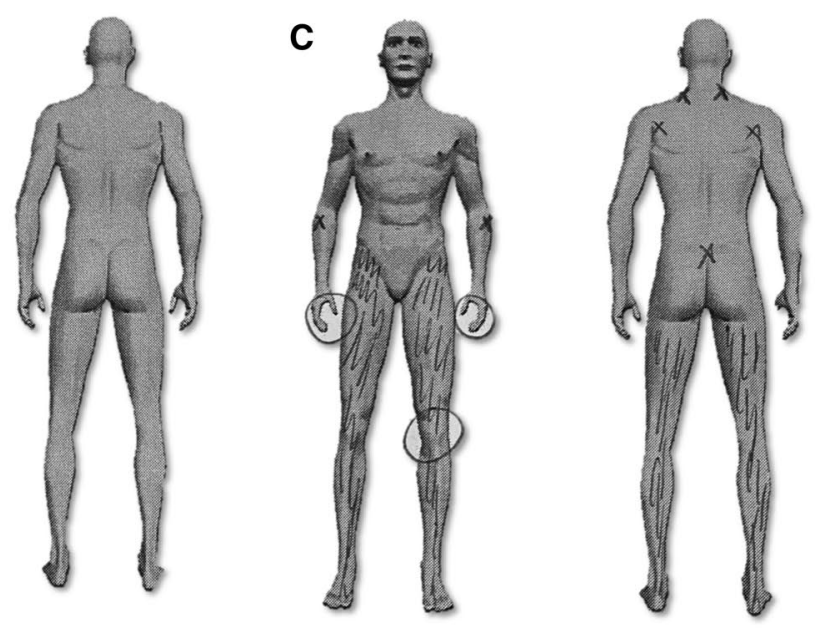

naire. Three of four categories were observed in our patients: A pain in one specific location, $\mathbf{B}$ pain in two separate limbs, and $\mathbf{C}$ pain diffusely spread throughout the body 
Table 1 General characteristics of the patient population

\begin{tabular}{|c|c|c|}
\hline Clinical characteristics & & $\begin{array}{l}\text { All patients } \\
\mathrm{N}=44\end{array}$ \\
\hline \multicolumn{3}{|l|}{ Demographic features } \\
\hline Sex (female) & & $25(56.8 \%)$ \\
\hline Age (years) & & $62.6 \pm 12.6$ \\
\hline Body mass index $\left(\mathrm{kg} / \mathrm{m}^{2}\right)^{*}$ & & $28.1 \pm 5.4$ \\
\hline \multicolumn{3}{|l|}{ Treatment features } \\
\hline $\begin{array}{l}\text { Duration of active disease } \\
\text { (years)* }\end{array}$ & & $8.2 \pm 6.7$ \\
\hline Duration of remission (years) $* *$ & & $\begin{array}{l}17.1(\mathrm{IQR} \\
7.3-25.4)\end{array}$ \\
\hline \multirow[t]{6}{*}{ Hypopituitarism } & & $17(38.6 \%)$ \\
\hline & GH deficiency & $9(20.5 \%)$ \\
\hline & Hypogonadism & $9(20.5 \%)$ \\
\hline & Hypocortisolism & $9(20.5 \%)$ \\
\hline & Hypothyroidism & $11(25.0 \%)$ \\
\hline & Diabetes insipidus & $4(9.1 \%)$ \\
\hline \multicolumn{3}{|l|}{ Type of treatment } \\
\hline & PharmaT & $5(11.4 \%)$ \\
\hline & Surgery & $18(40.9 \%)$ \\
\hline & Surgery + PharmaT & $13(29.5 \%)$ \\
\hline & Surgery + RT & $5(11.4 \%)$ \\
\hline & Surgery $+\mathrm{RT}+$ PharmaT & $2(4.5 \%)$ \\
\hline & RT + PharmaT & $1(2.3 \%)$ \\
\hline \multicolumn{3}{|l|}{ Biochemical characteristics } \\
\hline \multicolumn{3}{|l|}{$\mathrm{GH}(\mu \mathrm{g} / \mathrm{L}) \mathrm{GH}(\mu \mathrm{g} / \mathrm{L})$} \\
\hline & Pre-treatment $* * *$ & $\begin{array}{l}30.8(\mathrm{IQR} \\
15.0-53.5)\end{array}$ \\
\hline & Current $* * * *$ & $\begin{array}{l}1.4(\mathrm{IQR} \\
0.6-5.1)\end{array}$ \\
\hline \multicolumn{3}{|l|}{ IGF-1 (nmol/L) } \\
\hline & Pre-treatment $* * * *$ & $68.7 \pm 28.3$ \\
\hline & SDS**** & $6.5 \pm 2.9$ \\
\hline & Current* & $16.8 \pm 4.9$ \\
\hline & SDS* & $0.6 \pm 1.0$ \\
\hline
\end{tabular}

Data is reported as $\mathrm{N}(\%)$, mean $\pm \mathrm{SD}$, or median (IQR). Currently, eighteen patients received pharmaT; SMS analogue monotherapy $(\mathrm{N}=10)$, PegV monotherapy ( $=3)$, SMS analogue and PegV combination therapy $(\mathrm{N}=3)$, and SMS analogue and dopamine agonist combination therapy $(\mathrm{N}=2)$

$G H$ growth hormone, IGF-1 insulin-like growth factor-1, PegV Pegvisomant, PharmaT pharmacological treatment, $R T$ radiotherapy, $S M S$ somatostatin

*Data available in 40 patients

**Data available in 41 patients

****Data available in 21 patients

****Data available in 31 patients

therapy, and two patients were treated with SMS analogue and dopamine agonist combination therapy. Six patients (13.6\%) had diabetes mellitus. Ten patients $(22.7 \%)$
Table 2 Characteristics of neuropathic-like pain symptoms

\begin{tabular}{|c|c|c|}
\hline \multicolumn{2}{|c|}{ Neuropathic pain-like symptoms } & \multirow{2}{*}{$\begin{array}{l}\text { All patients } \\
\mathrm{N}=44\end{array}$} \\
\hline $\begin{array}{l}\text { Total painDETECT } \\
\text { score }\end{array}$ & & \\
\hline \multirow{3}{*}{$\begin{array}{l}\text { NP-like symptoms } \\
\text { categories }\end{array}$} & Likely & $4(9.1 \%)$ \\
\hline & Indeterminate & $6(13.6 \%)$ \\
\hline & Unlikely & $34(77.3 \%)$ \\
\hline \multicolumn{3}{|c|}{ Course of pain, radiation and localization } \\
\hline \multirow[t]{4}{*}{ Course of pain* } & $\begin{array}{l}\text { Continuous pain with slight } \\
\text { fluctuations }\end{array}$ & $14(40.0 \%)$ \\
\hline & $\begin{array}{l}\text { Continuous pain with pain } \\
\text { peaks }\end{array}$ & $4(11.4 \%)$ \\
\hline & $\begin{array}{l}\text { Pain-free periods with pain } \\
\text { peaks }\end{array}$ & $14(40.0 \%)$ \\
\hline & $\begin{array}{l}\text { Continuous pain with } \\
\text { extreme fluctuations }\end{array}$ & $3(8.6 \%)$ \\
\hline Radiating pain** & & $14(37.8 \%)$ \\
\hline \multirow[t]{4}{*}{ Pain localization*** } & One specific location & $8(25.8 \%)$ \\
\hline & One limb & $0(0.0 \%)$ \\
\hline & Two separate limbs & $16(51.6 \%)$ \\
\hline & $\begin{array}{l}\text { Diffusely spread throughout } \\
\text { the body }\end{array}$ & $7(22.6 \%)$ \\
\hline
\end{tabular}

Outcomes of the validated painDETECT questionnaire on neuropathic-like pain symptoms are shown, of which the total scores range from 0 to 35. Neuropathic-like pain symptom categories were unlikely-NP, total score $\leq 12$; indeterminate-NP, total score $13-18$; likely-NP, total score $\geq 19 \quad[43,44]$. Data is reported as $\mathrm{N}(\%)$, or median (IQR)

$I Q R$ interquartile range, $N P$ neuropathic pain

*Data available in 35 patients

**Data available in 37 patients

***Data available in 31 patients

currently used medication potentially affecting neuropathic pain (e.g., serotonin reuptake inhibitors, tricyclic antidepressants, pramipexol, sumatriptan or opioids).

\section{Pain}

Pain was reported by 35 patients $(79.5 \%)$, and median SF-36 pain component scores were 68.4 (IQR 57.1-89.8). Pain medication was used by 12 patients (i.e. paracetamol $\mathrm{N}=8$; nonsteroidal anti-inflammatory drugs $\mathrm{N}=4$ ). Median painDETECT total scores were 5 (IQR 2-12), as shown in Table 2. Based on painDETECT total scores, four patients (9.1\%) likely suffered from neuropathic-like pain symptoms, six patients $(13.6 \%)$ indeterminately suffered from neuropathic-like pain symptoms, and 34 patients $(77.3 \%)$ were unlikely to suffer from neuropathic pain. All four patients with likely-NP were female $(\mathrm{P}=0.186)$. 
Three of four likely-NP patients suffered from relevant comorbidities: demyelinating polyneuropathy $(\mathrm{N}=1)$, diabetes mellitus $(\mathrm{N}=1)$, and spondylolisthesis $(\mathrm{N}=1)$. Of the six patients with indeterminate neuropathic-like pain symptoms, two patients suffered from a disc herniation, one patient had diabetes mellitus, and one patient had multiple sclerosis. Twelve unlikely-NP patients suffered from relevant comorbidities (viz. diabetes mellitus, polyneuropathy, carpal tunnel syndrome, brachial plexopathy, disc herniation, neural foraminal stenosis, spinal cord injury). When assessing whether the locations of pain from these comorbidities overlapped with the regions of pain indicated on the painDETECT questionnaire, overlap of the painful regions was observed in four patients (total $\mathrm{N}=1$, and partial $\mathrm{N}=3$ ), whereas no overlap was observed in three patients.

Out of the ten indeterminate, and likely NP patients, four patients used medication potentially affecting neuropathic pain-like symptoms: two likely-NP patients (SSRI prescribed for depression $(\mathrm{N}=1)$, amitriptyline prescribed for neuropathic pain caused by demyelinating polyneuropathy), and two indeterminate-NP patients (SSRIs prescribed for depression $(\mathrm{N}=2))$.

\section{Relationship between painDETECT, self-reported joint symptoms and QoL}

Higher painDETECT scores were correlated with lower HR-QoL (PCS: $r=-0.46, P=0.003$; MCS: $r=-0.37$, $\mathrm{P}=0.018$, respectively), especially with the pain component scores $(r=-0.63, P<0.0001)$, as depicted in Fig. 2 . In addition, higher painDETECT scores were associated with worse WOMAC $(\mathrm{r}=0.56, \mathrm{P}<0.0001)$, AUSCAN $(r=0.62, P<0.0001)$, and DASH scores $(r=0.63$, $\mathrm{P}<0.0001$ ) (Fig. 2).

\section{Risk factors for neuropathic pain}

The effects of age, sex and BMI, all being factors known to influence neuropathic pain in the general population [17], were assessed, and solely female sex was significantly associated with neuropathic-pain like symptoms $(\beta=5.24 \pm 2.22, P=0.024)$. Following adjustment for sex, painDETECT scores were not associated with pre-treatment IGF-1 levels $(\beta=-0.05 \pm 0.05, P=0.344)$, current GH levels $(\beta=-0.00 \pm 0.01, P=0.586)$, treatment modality (viz. surgery and radiotherapy $v s$ pharmacological
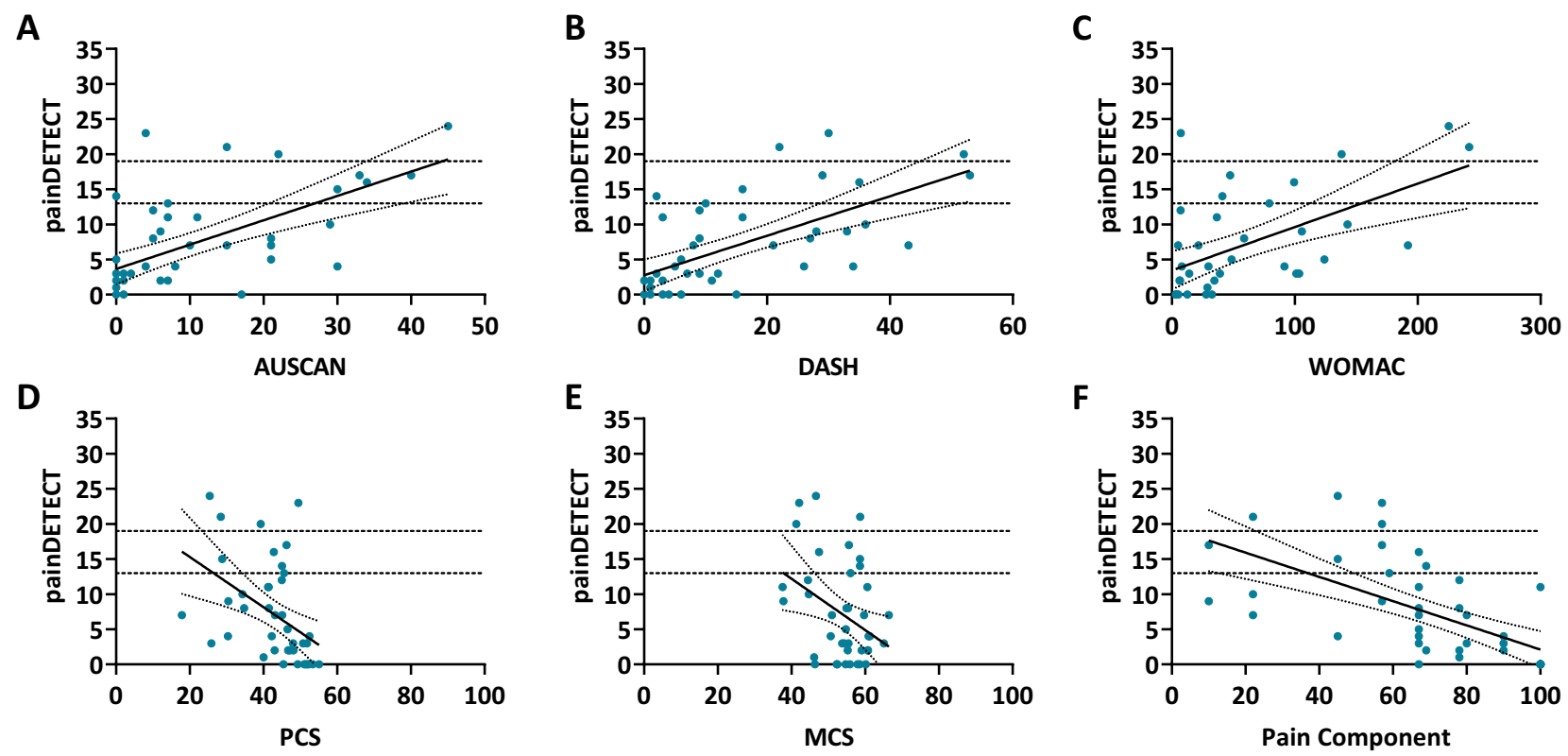

Fig. 2 Correlations between painDETECT scores and OA-related disability and HR-QoL. Correlations between painDETECT scores and AUSCAN, DASH, WOMAC, MCS, PCS, and pain component scores are depicted. Horizontal dashed lines at painDETECT values 13 and 19 indicating indeterminate and likely, respectively, neuropathic-like pain symptoms cut-off points. A Correlation between painDETECT and AUSCAN scores $(\mathrm{r}=0.62, \mathrm{P}<0.0001)$, $\mathrm{B}$ correlation between painDETECT and DASH scores $(\mathrm{r}=0.63, \mathrm{P}<0.0001)$, $\mathrm{C}$ correlation between painDETECT and WOMAC scores $(r=0.56, \mathrm{P}<0.0001)$, D

correlation between painDETECT and PCS $(r=-0.46, \mathrm{P}=0.003), \mathbf{E}$ correlation between painDETECT and MCS $(\mathrm{r}=-0.37, \mathrm{P}=0.018)$, F correlation between painDETECT and pain component score $(\mathrm{r}=-0.63, \mathrm{P}<0.0001)$. AUSCAN Australian/Canadian Osteoarthritis Hand Index, DASH Disabilities of the Arm, Shoulder and Hand, WOMAC Western Ontario and McMaster Universities Arthritis Index, $M C S$ mental health component scores, PCS physical health component score 
treatment $)(\beta=-0.40 \pm 2.05, \mathrm{P}=0.848)$, active disease duration $(\beta=0.21 \pm 0.16, P=0.192)$, remission duration $(\beta=0.03 \pm 0.10, P=0.770)$, or hypopituitarism $(\beta=2.72 \pm 2.20, \mathrm{P}=0.223)$.

\section{Pain radiation and localization}

Radiation of pain could be assessed in 37 patients (Table 2). Radiating pain was present in 3 out of 4 patients with likelyNP, 2 out of 5 patients with indeterminate-NP, and 9 out of 28 patients with unlikely-NP patients, respectively. Course of pain could be assessed in 35 patients (Table 2). Three out of 4 patients with likely-NP patients experienced continuous pain with extreme fluctuations. Indeterminate-, and unlikelyNP patients reported generally pain-free periods with pain peaks, or continuous pain with slight fluctuations.

In addition, 31 patients provided drawings to assess pain localization. None of the patients reported pain in one limb (category 2), whereas examples of the other categories are shown in Fig. 1. Twenty-eight of 31 patients localizing pain on the painDETECT questionnaire also reported the presence of pain on the HR-QoL questionnaire. In these 28 patients, pain was localized in one specific site in 6 patients, in two separate limbs in 15 patients, and diffusely spread throughout the body in 7 patients. The remaining 3 patients that did not report pain on the HR-QoL questionnaire, indicated some form of pain in either one specific location $(\mathrm{N}=2)$, or two separate limbs $(\mathrm{N}=1)$.

\section{Discussion}

The present explorative, questionnaire-based study reported that, despite a high prevalence of pain in controlled acromegaly patients, neuropathic-like pain symptoms are a relatively infrequent finding, and occurred in females only. Patients with higher painDETECT scores, reflecting a higher probability of neuropathic-like pain symptoms, reported lower HR-QoL, and more OA-related disability. All but two patients with likely or indeterminate neuropathic-like pain symptoms were diagnosed with relevant comorbidities. Apart from female sex, no other (acromegaly-specific) factors were associated with neuropathic-like pain symptoms in our cohort.

Neuropathic pain is caused by altered sensory signal transduction to the brain due to lesions or diseases of the somatosensory nervous system. Symptoms typically include burning, tingling sensations (dysesthesia), and pain upon non-painful stimuli (allodynia) [55]. In the general population, neuropathic-like pain symptoms are more frequent in women (8.0\% in females vs $5.7 \%$ in males), and individuals $>50$ years of age [17], which strengthens our findings that reported neuropathic-like pain symptoms were worse, and experienced specifically in female acromegalics. Notably, the increased reporting of pain in females is not specific for neuropathic pain, since females report more pain in general, including OA-related pain $[56,57]$.

In the present study, some form of pain was self-reported by over $75 \%$ of patients with controlled acromegaly, which is in line with previously reported prevalence [3]. However, although pain is a prevalent symptom in acromegaly, neuropathic-like pain symptoms were likely in only $10 \%$ of patients, and potentially observed in $25 \%$ of patients (i.e. likely-NP in $10 \%$, and indeterminate-NP in $15 \%$ of patients). The prevalence of neuropathic-like pain symptoms in our population is slightly higher than the sole other study on neuropathic pain in pituitary adenoma patients, reporting that neuropathic pain was likely in approximately $5 \%$ of acromegaly patients using the same painDETECT questionnaire [3]. In addition to the aforementioned study, two case reports have reported on the invalidating potential of (severe) neuropathic pain in acromegaly [20,58], highlighting the need for additional studies on this topic. The prevalence of neuropathic pain in our study among long-term controlled acromegaly patients was comparable with the general population (7 to 10\%) [59], which was quite unexpected as acromegaly is associated with multiple typical neuropathic pain conditions, e.g. diabetic neuropathy, and compression neuropathies [4, 7, 8, 19-21]. Moreover, acromegaly patients have a high prevalence of arthropathy that partly resembles primary $\mathrm{OA}$, and it is known from studies among patients with knee and hip OA that the prevalence of neuropathiclike pain symptoms is $23 \%$ [60]. The controlled status of included acromegaly patients might partially explain the low prevalence of neuropathic-like pain symptoms in our controlled patient population, since treatment was shown to significantly decrease neuropathic symptoms (e.g. paresthesia) in a meta-analysis of 24 studies [2].

Furthermore, we assessed the localization of pain, and presence of pain radiation. The localization of pain was limited to one specific location or joint in $25 \%$ of patients, with $75 \%$ patients localizing their pain in multiple limbs and joints, or present diffusely throughout the entire body. Furthermore, radiating pain - a phenomenon whereby pain of one specific origin is perceived in other locations-was observed in approximately $40 \%$ of patients. Since radiating pain is one of the characteristics of neuropathic pain, presence of pain radiation might underline the existence of, or risk to develop neuropathic pain in the future.

Higher upper and lower limb disability scores, and lower mental and physical HR-QoL were observed in patients with (potential) neuropathic-like pain symptoms. These observations are in line with general population studies showing that neuropathic pain is generally more invalidating than nociceptive pain $[17,18]$, and is thereby significantly associated 
with depression and poorer HR-QoL $[3,18]$. The sole risk factor for neuropathic-like pain symptoms in our study with controlled acromegaly patients was female sex, whilst age, and BMI (factors known to influence neuropathic pain in the general population [17]) were not observed in the current patient population. Measures for acromegalic disease activity (e.g. GH and IGF-1 levels, and disease duration) did not influence the risk of neuropathic-like pain symptoms. Therefore, we assume that neuropathic pain and neuropathic-like pain symptoms in acromegaly are likely to be caused by acromegalic comorbidities (e.g. neuro-toxic metabolic dysregulation, or compression neuropathies), and not directly by GH and IGF-1 excess. Moreover, the presence of neuropathic-like pain symptoms could be explained by neurological comorbidities not associated with acromegaly, e.g. disc herniation, and multiple sclerosis [61].

Based on the findings of our study, and the scarcely available literature on neuropathic pain in the general population, we have constructed an algorithm for the diagnostics and management of pain in acromegaly, summarized in Fig. 3. In the case of a patient with pain, or OA-related disability, we advise to assess disease activity, and presence of associated comorbidities (e.g. OA, compression neuropathies, and vertebral fractures). Moreover, we advise to assess neuropathic-like pain symptoms using a self-administered questionnaire in patients to determine the likelihood of neuropathic and nociceptive pain, and to ensure adequate pain treatment, as neuropathic pain requires specific analgesic therapy with anticonvulsants and tricyclic antidepressants $[22,23]$.

Several limitations need to be addressed. Notably, outcomes of the SF-36 pain component and painDETECT questionnaire were partially non-overlapping, resulting in a potential higher prevalence of pain of over $85 \%$ in the present population. Moreover, as the study cohort was small, numerical questionnaire outcomes were used for analyses, whereas the painDETECT questionnaire was originally developed to have a categorical outcome. Additionally, presence of neuropathic-like pain symptoms was solely determined based on the painDETECT questionnaire, as clear, objective criteria for identifying neuropathic pain remain unavailable. Furthermore, the origin of neuropathic pain in acromegaly is difficult to determine, as multiple painful complications may coexist. Lastly, the present patient population included controlled acromegaly patients only, whereas patients with active disease might be more severely affected with regards to neuropathic (pain) symptoms.

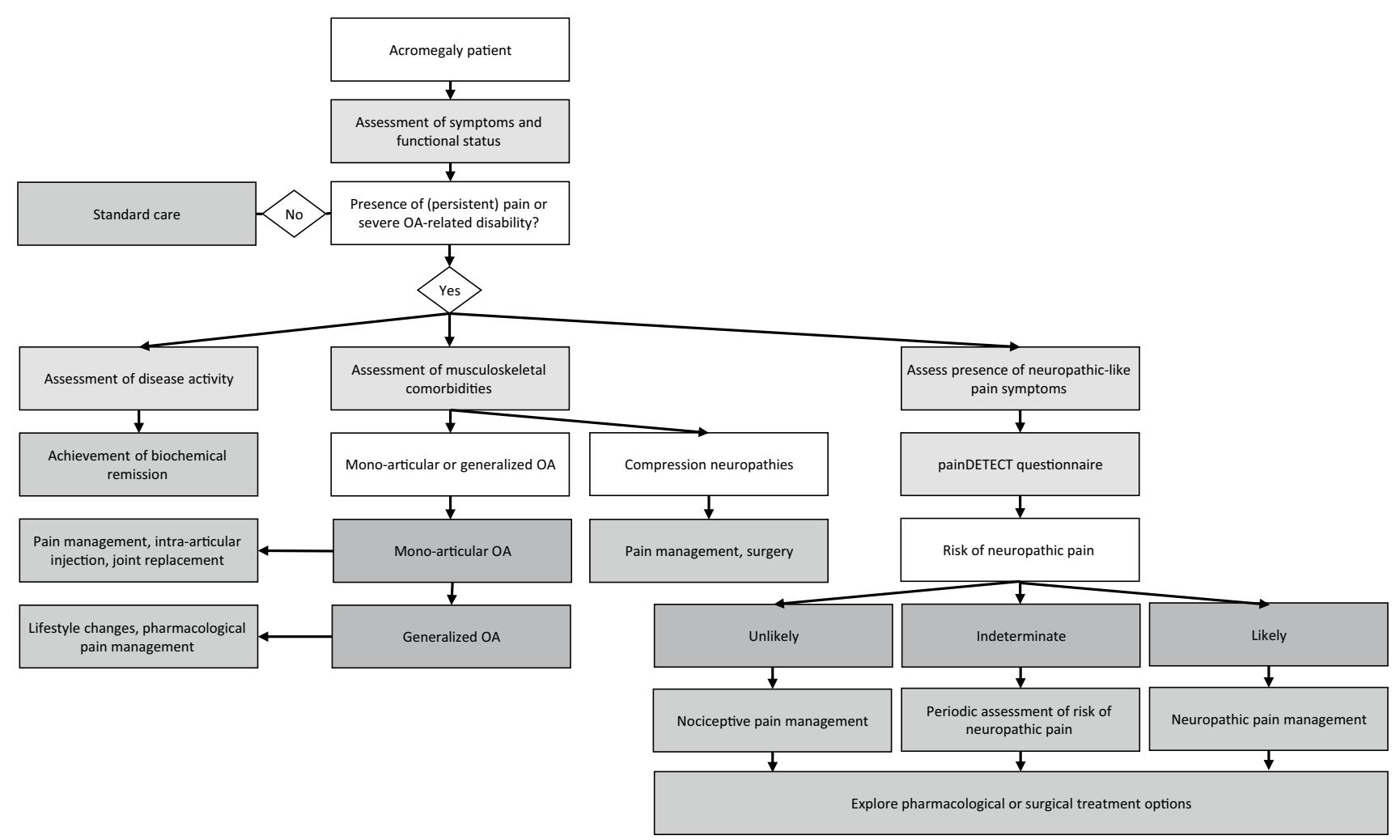

Fig. 3 Flowchart of decision-making regarding pain diagnostics and management in acromegaly patients. Proposed algorithm for the diagnosis, and treatment for pain in acromegaly, including the assessment of the presence of neuropathic $=$ like pain symptoms using the painDETECT questionnaire. $O A$ osteoarthritis 
In conclusion, although pain is a prevalent symptom in acromegaly patients, the infrequently occurring neuropathiclike pain symptoms - only found in females-suggests that pain in controlled acromegaly mostly is of nociceptive nature. The presence of neuropathic-like pain symptoms was associated with lower mental and physical HR-QoL and more OA-related disability. We advise to assess for neuropathic-like pain symptoms in patients with compromised HR-QoL, and severe, persisting pain despite regular pain medication.

Supplementary Information The online version contains supplementary material available at https://doi.org/10.1007/s11102-021-01190-z.

Funding Not applicable.

Data availability Available upon request.

Code availability Not applicable.

\section{Declarations}

Conflict of interest Not applicable.

Ethical approval All procedures performed in these studies involving human participants (i.e. patients) were in accordance with the ethical standards of the institutional research committee, and with the 1964 Helsinki Declaration and its later amendments. The studies were approved by the Medical Ethics Committee of the Leiden University Medical Center (LUMC; identification number NL58163.058.16).

Consent to participate All patients gave written informed consent prior to participation in the studies.

Open Access This article is licensed under a Creative Commons Attribution 4.0 International License, which permits use, sharing, adaptation, distribution and reproduction in any medium or format, as long as you give appropriate credit to the original author(s) and the source, provide a link to the Creative Commons licence, and indicate if changes were made. The images or other third party material in this article are included in the article's Creative Commons licence, unless indicated otherwise in a credit line to the material. If material is not included in the article's Creative Commons licence and your intended use is not permitted by statutory regulation or exceeds the permitted use, you will need to obtain permission directly from the copyright holder. To view a copy of this licence, visit http://creativecommons.org/licenses/by/4.0/.

\section{References}

1. Wassenaar M et al (2010) Clinical osteoarthritis predicts physical and psychological QoL in acromegaly patients. Growth Hormon IGF Res 20:226-233

2. Broersen LHA et al (2021) Improvement in symptoms and healthrelated quality of life in acromegaly patients: a systematic review and meta-analysis. J Clin Endocrinol Metab 106(2):577-587

3. Dimopoulou C et al (2014) Clinical characteristics of pain in patients with pituitary adenomas. Eur J Endocrinol 171(5):581-591
4. Chanson P, Salenave S (2008) Acromegaly. Orphanet J Rare Dis $3: 17$

5. Vilar L et al (2017) Acromegaly: clinical features at diagnosis. Pituitary 20(1):22-32

6. Kyriakakis $N$ et al (2017) Impaired quality of life in patients with treated acromegaly despite long-term biochemically stable disease: Results from a 5-years prospective study. Clin Endocrinol (Oxf) 86(6):806-815

7. Kameyama $S$ et al (1993) Subclinical carpal tunnel syndrome in acromegaly. Neurol Med Chir (Tokyo) 33(8):547-551

8. Abreu A et al (2016) Challenges in the diagnosis and management of acromegaly: a focus on comorbidities. Pituitary 19(4):448-457

9. Claessen KM et al (2012) Progression of acromegalic arthropathy despite long-term biochemical control: a prospective, radiological study. Eur J Endocrinol 167(2):235-244

10. Wassenaar MJ et al (2011) High prevalence of vertebral fractures despite normal bone mineral density in patients with long-term controlled acromegaly. Eur J Endocrinol 164(4):475-483

11. Claessen KM et al (2014) Increased clinical symptoms of acromegalic arthropathy in patients with long-term disease control: a prospective follow-up study. Pituitary 17(1):44-52

12. Giustina A (2020) Acromegaly and vertebral fractures: facts and questions. Trends Endocrinol Metab 31(4):274-275

13. Pelsma ICM et al (2020) Progression of vertebral fractures in long-term controlled acromegaly: a 9-year follow-up study. Eur J Endocrinol. https://doi.org/10.1530/endoabs.70.AEP215

14. Mazziotti $\mathrm{G}$ et al (2015) Bone turnover, bone mineral density, and fracture risk in acromegaly: a meta-analysis. J Clin Endocrinol Metab 100:384-394

15. Jadresic A et al (1982) The acromegaly syndrome. Relation between clinical features, growth hormone values and radiological characteristics of the pituitary tumours. Q J Med 51(202):189-204

16. IASP Terminology. 1994 December 14, 2017 [cited 2020 October, 15]; https://www.iasp-pain.org/terminology?navItemNumber= 576.

17. Bouhassira D et al (2008) Prevalence of chronic pain with neuropathic characteristics in the general population. Pain 136(3):380-387

18. Smith BH et al (2007) Health and quality of life associated with chronic pain of predominantly neuropathic origin in the community. Clin J Pain 23(2):143-149

19. Chanson P et al (2009) Pituitary tumours: acromegaly. Best Pract Res Clin Endocrinol Metab 23(5):555-574

20. Kopsky DJ, KeppelHesselink JM (2013) Neuropathic pain as a result of acromegaly, treated with topical baclofen cream. J Pain Symptom Manage 46(4):4-5

21. Hannon AM, Thompson CJ, Sherlock M (2017) Diabetes in patients with acromegaly. Curr Diab Rep 17(2):8

22. Finnerup NB, Sindrup SH, Jensen TS (2010) The evidence for pharmacological treatment of neuropathic pain. Pain 150(3):573-581

23. Ohtori S et al (2013) Efficacy of combination of meloxicam and pregabalin for pain in knee osteoarthritis. Yonsei Med J 54(5):1253-1258

24. Pelsma ICM et al (2020) Progression of acromegalic arthropathy in long-term controlled acromegaly patients: 9 years of longitudinal follow-up. J Clin Endocrinol Metab 106:188

25. Biermasz NR et al (2012) Automated image analysis of hand radiographs reveals widened joint spaces in patients with long-term control of acromegaly: relation to disease activity and symptoms. Eur J Endocrinol 166(3):407

26. Wassenaar MJ et al (2011) Arthropathy in long-term cured acromegaly is characterised by osteophytes without joint space narrowing: a comparison with generalised osteoarthritis. Ann Rheum Dis 70(2):320-325 
27. Wassenaar MJ et al (2009) The exon-3 deleted growth hormone receptor polymorphism predisposes to long-term complications of acromegaly. J Clin Endocrinol Metab 94(12):4671-4678

28. Wassenaar MJ et al (2009) High prevalence of arthropathy, according to the definitions of radiological and clinical osteoarthritis, in patients with long-term cure of acromegaly: a casecontrol study. Eur J Endocrinol 160(3):357-365

29. Wassenaar MJ et al (2010) Acromegaly is associated with an increased prevalence of colonic diverticula: a case-control study. J Clin Endocrinol Metab 95(5):2073-2079

30. Claessen KM et al (2013) Two phenotypes of arthropathy in longterm controlled acromegaly? A comparison between patients with and without joint space narrowing (JSN). Growth Horm IGF Res 23(5):159-164

31. Claessen KMJA et al (2017) Acromegalic arthropathy in various stages of the disease: an MRI study. Eur J Endocrinol 176(6):779

32. van der Klaauw A et al (2008) Disease-specific impairments in quality of life during long-term follow-up of patients with different pituitary adenomas. Clin Endocrinol 69:775-784

33. Ross HA et al (2014) Harmonization of growth hormone measurement results: the empirical approach. Clin Chim Acta 432:72-76

34. Cole TJ (1990) The LMS method for constructing normalized growth standards. Eur J Clin Nutr 44(1):45-60

35. Rikken B et al (1998) Plasma levels of insulin-like growth factor (IGF)-I, IGF-II and IGF-binding protein-3 in the evaluation of childhood growth hormone deficiency. Horm Res 50(3):166-176

36. Rienstra $W$ et al (2015) The modified painDETECT questionnaire for patients with hip or knee osteoarthritis: translation into Dutch, cross-cultural adaptation and reliability assessment. PLoS ONE 10(12): 0146119

37. De Andrés J et al (2012) Cultural adaptation and validation of the painDETECT scale into Spanish. Clin J Pain 28(3):243-253

38. Alkan $\mathrm{H}$ et al (2013) Turkish version of the painDETECT questionnaire in the assessment of neuropathic pain: a validity and reliability study. Pain Med 14(12):1933-1943

39. Matsubayashi $Y$ et al (2013) Validity and reliability of the Japanese version of the painDETECT questionnaire: a multicenter observational study. PLoS ONE 8(9):68013

40. Gudala K, Ghai B, Bansal D (2017) Neuropathic pain assessment with the painDETECT questionnaire: cross-cultural adaptation and psychometric evaluation to Hindi. Pain Pract 17(8):1042-1049

41. Sung JK et al (2017) Korean version of the painDETECT questionnaire: a study for cultural adaptation and validation. Pain Pract 17(4):494-504

42. Cappelleri JC et al (2015) A cross-sectional study examining the psychometric properties of the painDETECT measure in neuropathic pain. J Pain Res 8:159-167

43. Freynhagen $\mathrm{R}$ et al (2016) The painDETECT project: far more than a screening tool on neuropathic pain. Curr Med Res Opin 32(6):1033-1057

44. Freynhagen R et al (2006) PainDETECT: a new screening questionnaire to identify neuropathic components in patients with back pain. Curr Med Res Opin 22(10):1911-1920

45. Bellamy $\mathrm{N}$ et al (2002) Clinimetric properties of the AUSCAN Osteoarthritis Hand Index: an evaluation of reliability, validity and responsiveness. Osteoarthritis Cartilage 10(11):863-869
46. Khan WS et al (2008) The 'M2 DASH'-Manchester-modified disabilities of arm shoulder and hand score. Hand (N Y) 3(3):240-244

47. Beaton DE et al (2001) Measuring the whole or the parts? Validity, reliability, and responsiveness of the disabilities of the arm, shoulder and hand outcome measure in different regions of the upper extremity. J Hand Ther 14(2):128-146

48. Solway SBD, McConnell S, Bombardier C (2002) The DASH outcome measure user's manual

49. Woolacott NF, Corbett MS, Rice SJ (2012) The use and reporting of WOMAC in the assessment of the benefit of physical therapies for the pain of osteoarthritis of the knee: findings from a systematic review of clinical trials. Rheumatology (Oxford) 51(8):1440-1446

50. Veehof MM et al (2002) Psychometric qualities of the Dutch language version of the disabilities of the arm, shoulder, and hand questionnaire (DASH-DLV). J Hand Ther 15(4):347-354

51. Bellamy $\mathrm{N}$ et al (1988) Validation study of WOMAC: a health status instrument for measuring clinically important patient relevant outcomes to antirheumatic drug therapy in patients with osteoarthritis of the hip or knee. J Rheumatol 15(12):1833-1840

52. Groningen R (2012) Het meten van de algemene gezondheidstoestand met de Rand-36:24-28

53. Ware JE (1993) SF-36 Health Survey Manual and Interpretation Guide. The Health Institute, New England Medical Center - Boston

54. Jenkinson C, Coulter A, Wright L (1993) Short form 36 (SF36) health survey questionnaire: normative data for adults of working age. BMJ 306(6890): 1437-1440

55. Colloca L et al (2017) Neuropathic pain. Nat Rev Dis Primers 3:17002

56. Fu K, Robbins SR, McDougall JJ (2018) Osteoarthritis: the genesis of pain. Rheumatology (Oxford) 57(suppl_4):iv43-iv50

57. Hunter DJ, Bierma-Zeinstra S (2019) Osteoarthritis. Lancet 393(10182):1745-1759

58. Naccache DD et al (2014) Diabetic neuropathic cachexia: a case report. J Med Case Rep 8:20

59. van Hecke $\mathrm{O}$ et al (2014) Neuropathic pain in the general population: a systematic review of epidemiological studies. Pain 155(4):654-662

60. French HP, Smart KM, Doyle F (2017) Prevalence of neuropathic pain in knee or hip osteoarthritis: a systematic review and metaanalysis. Semin Arthritis Rheum 47(1):1-8

61. Baron R, Binder A, Wasner G (2010) Neuropathic pain: diagnosis, pathophysiological mechanisms, and treatment. Lancet Neurol 9(8):807-819

Publisher's Note Springer Nature remains neutral with regard to jurisdictional claims in published maps and institutional affiliations. 